\title{
Appropriation foncière et bourgeoisie marchande : éléments pour une analyse de l'économie marchande du Bas-Canada avant 1846
}

\section{Gérald Bernier et Daniel Salée}

Volume 36, numéro 2, septembre 1982

URI : https://id.erudit.org/iderudit/304046ar

DOI : https://doi.org/10.7202/304046ar

Aller au sommaire du numéro

Éditeur(s)

Institut d'histoire de l'Amérique française

ISSN

0035-2357 (imprimé)

1492-1383 (numérique)

Découvrir la revue

Citer cet article

Bernier, G. \& Salée, D. (1982). Appropriation foncière et bourgeoisie marchande : éléments pour une analyse de l'économie marchande du Bas-Canada avant 1846. Revue d'histoire de l'Amérique française, 36(2), 163-194. https://doi.org/10.7202/304046ar d'utilisation que vous pouvez consulter en ligne. 


\title{
APPROPRIATION FONCIÈRE ET BOURGEOISIE MARCHANDE: ÉLÉMENTS POUR UNE ANALYSE DE L'ÉCONOMIE MARCHANDE DU BAS-CANADA AVANT 1846*
}

\author{
GÉRALD BERNIER \\ Département de science politique \\ Université de Montréal \\ DANIEL SALÉE \\ Département de science politique \\ Université York
}

\begin{abstract}
Malgré une littérature relativement abondante sur l'histoire du Bas-Canada, on est forcé de noter une certaine carence au niveau de l'étude de l'économie politique. L'analyse procède souvent à partir de formules ou d'interprétations consacrées, sans dépasser les apparences formelles. Ainsi on prend pour acquis et on remet rarement en question la nature capitaliste de la formation sociale bas-canadienne. Il existe dans l'historiographie actuelle sur le Bas-Canada une tendance marquée à présenter ou à concevoir les activités économiques de la «bourgeoisie marchande» 1 et, partant, l'ensemble de la société bas-canadienne, comme étant typi-
\end{abstract}

* Cette recherche a bénéficié de l'appui financier du fonds FCAC du Ministère de l'Éducation du Québec. Nous désirons remercier Jocelyne Dion-Clermont et Gisèle Mainville qui ont dactylographié le manuscrit.

Le terme bourgeoisie marchande fait ici référence à la classe sociale qui, à partir de ses activités commerciales et de l'exercice de son monopole à l'intérieur des échanges coloniaux pendant toute la première moitié du 19e siècle, impose sur l'ensemble de la formation sociale bas-canadienne sa domination économique et détermine en grande partie l'orientation de la structure économique. L'emploi du terme bourgeoisie nous apparaît comme un compromis: nous sommes conscients que la bourgeoisie, prise dans son sens strict, est surtout un produit du capitalisme dans sa phase industrielle et opère à l'intérieur de la sphère de production. Qu'il soit entendu que notre recours au terme bourgeoisie marchande constitue ici une convention de langage qui recouvre en fait la classe de marchands et de financiers qui parvient à réaliser une certaine accumulation de capital et à accroître ses capacités de thésauriser. Notons au passage que cette bourgeoisie marchande au BasCanada n'est pas homogène. Elle comporte deux groupes. Le premier, le plus influent aux plans économique et politique, axe avant tout ses activités dans la sphère d'échange avec la métropole. Le second déploie l'essentiel de ses activités commerciales sur le marché intérieur et dépend, en l'occurrence, du développement des potentialités locales de la sphère de production. Sur la base des données que nous possédons, les comportements attribués à la bourgeoisie marchande dans le cadre de ce texte semblent, à première vue, davantage le fait du premier groupe. Seule une recherche plus approfondie appuyée sur une analyse empirique des activités économiques d'un large échantillon des marchands oeuvrant à cette époque permettrait d'asseoir cette hypothèse sur des bases plus solides. Cela est d'ailleurs confirmé pour une période subséquente par Gérald Tulchinsky «The Montreal Business Community, 1837-1853» in David S. MacMillan (sous la direction de), Canadian Business History (Toronto, Mc Clelland et Stewart, 1972), 125-143. 
quement capitalistes. Il est vrai qu'entre 1807 et 1846 l'essor du commerce du bois, en particulier, donnera le jour à des fortunes considérables; l'origine de certaines grandes dynasties capitalistes canadiennes remonte même aux premières années du 19e siècle. Pour cette raison, il peut être tentant d'appréhender l'ensemble des activités de la bourgeoisie marchande comme le fait d'une classe capitaliste au sens propre.

Pourtant, nous croyons devoir soutenir ici que la nature des pratiques de la bourgeoisie marchande et de la structure économique qu'elles déterminent n'autorise pas à les présenter comme étant capitalistes stricto sensu.

En fait, l'attitude analytique, partagée à la fois par l'historiographie libérale canadienne et certains auteurs se réclamant du marxisme ${ }^{2}$, et qui consiste à considérer la bourgeoisie marchande comme une classe sociale capitaliste, constitue une méprise. Ce n'est pas parce que du point de vue économique, cette classe sociale reste essentiellement préoccupée par la consolidation du système d'échange, et qu'elle réalise une certaine accumulation de capital, qu'elle peut être désignée d'emblée comme étant capitaliste. Marx a démontré que ce n'est que dans le processus historique de transformation de la force de travail en marchandise (salariat), et dans l'accumulation de capital à des fins productives, que s'inscrit l'origine du développement du capitalisme. Le marché ne fournit pas à lui seul l'élan nécessaire à la mise en branle de la dynamique capitaliste ${ }^{3}$. Dans le capitalisme, la relation économique fondamentale est déterminée par l'obligation dans laquelle se trouve le travailleur (producteur direct) de vendre sa force de travail au propriétaire des moyens de production. Leur rapport est d'abord circonscrit par les impératifs de la production et non pas de l'échange.

Sa négligence à développer de manière significative la sphère de production avant la seconde moitié du 19e siècle disqualifie la bourgeoisie marchande comme classe du mode de production capitaliste (MPC). Plusieurs auteurs se sont déjà penchés sur la problématique que pose la nature réelle du capital marchand et d'une classe de marchands dans leur articulation au MPC ainsi qu'à d'autres modes de production. Leurs conclusions convergent: l'activité marchande sous ses diverses facettes (accumulation,

2 Voir en particulier les travaux des historiens Fernand Ouellet et Donald Creighton sur cette période. Voir aussi les travaux plus théoriques des penseurs marxistes tels que I. Wallerstein et A. Gunder Frank.

R. Brenner, "The Origins of Capitalist Development: A Critique of NeoSmithian Marxism», New Left Review, 104 (juillet-août 1977), section III. 
échange, etc.) ne relève pas du MPC. Trop souvent a-t-on confondu capital et capitalisme ${ }^{4}$. Louis Althusser résume bien la question lorsqu'il remarque avec justesse:

De ce que l'économie industrielle est sortie, à un moment donné, de l'accumulation dont l'économie marchande constitua un moment, on tire trop souvent la conclusion que l'économie marchande était dans son principe étrangère à la société féodale. Rien n'est plus douteux. Il suffit alors de voir dans quel sens jouait cette économie marchande pour conclure qu'elle était une pièce assez bien intégrée du système féodal lui-même: le mercantilisme est justement la politique et la théorie de cette intégration. ${ }^{5}$

L'importance et la domination économique de la bourgeoisie marchande au Bas-Canada ont été suffisamment démontrées (cf. Creighton, Ouellet, Naylor) pour nous permettre d'affirmer que la structure économique bas-canadienne de la première moitié du $19 \mathrm{e}$ siècle constitue une économie marchande. Économie dont les traits essentiels sont:

1. une hypertrophie de la sphère de la circulation au détriment de la sphère de production; 2 . la domination d'un capital accumulé et remis en circulation dans la sphère d'échange (capital marchand); 3. l'exploitation foncière à des fins spéculatives et/ou la mise en valeur de la terre dans une optique mercantiliste (les catégories de biens produits étant déterminées par une demande extérieure); 4. la reproduction élargie de rapports de production non capitalistes dominants.

Ce texte sera consacré plus particulièrement à l'examen des deux derniers aspects de ce type d'économie. On verra qu'il existe une espèce de symbiose entre le régime seigneurial caractérisé par la dominance de rapports de production de type féodal et l'activité économique des marchands qui, loin de s'en trouver gênée, contribue à la reproduction des dits rapports. Pour étayer la démonstration, on portera l'analyse sur le terrain des pratiques foncières de la bourgeoisie marchande bas-canadienne au cours de la première moitié du 19e siècle. Le choix de cet objet d'analyse n'est pas fortuit, mais tient au fait que, dans une formation sociale où l'exploitation de l'agriculture et des potentialités foncières en général

4 Voir entre autres Maurice Dobb, Études sur le développement du capitalisme (Paris, Maspéro, Collections «Les textes à l'appui», 1969), 18; Ernesto Laclau, Ideology, Politics and Marxism (Londres, New Left Books, 1977), 26; Henry Veltmeyer, «A Central Issue in Dependency Theory», Revue canadienne de sociologie et d'anthropologie, 17, 3 (1980): 198-213.

Louis Althusser, Montesquieu, la politique et l'histoire (Paris, Presses universitaires de France, 1959, 1974), 113-114. 
constitue l'activité essentielle de la majorité de la population, la propriété de la terre est obligatoirement un facteur décisif d'organisation socio-économique. Par conséquent, les relations de propriété/non-propriété foncière vont déterminer au premier chef les contours particuliers des rapports sociaux. Aussi, ces rapports qui s'inscrivent dans la propriété foncière ont un impact prédominant sur tout l'édifice social; l'existence de ces rapports sociaux de nature féodale imprègne de manière dominante toutes les structures de la formation sociale.

De façon générale, l'objectif de cet article est de démontrer que l'économie marchande et ses agents sociaux dominants, en dépit de leur allure apparemment capitaliste, procèdent d'une logique de nature fondamentalement féodaliste (ou non-capitaliste). En ce sens, nous entendons contribuer à la présentation d'éléments préliminaires susceptibles de permettre une appréciation nouvelle de l'économie politique bas-canadienne.

La période 1807-1846 circonscrit le champ temporel de l'étude. La première année coïncide avec l'entrée du bois dans le circuit d'échange avec la métropole. Ce fait contribue à engendrer un mouvement de spéculation foncière, porté par la bourgeoisie marchande, et qui gênera l'accès à la propriété foncière pour les masses paysannes autochtones et les immigrants britanniques. L'adhésion de la Grande-Bretagne au libre-échange en 1846 annonce le déclin de l'économie marchande et l'amorce d'un développement plus diversifié, axé notamment sur la sphère de production.

\section{I - Ethnie, bourgeoisie marchande et appropriation foncière: à propos d'un mythe}

En matière d'appropriation foncière au Bas-Canada, deux questions retiennent l'attention de l'historiographie: 1) le caractère prétendument ethnique de l'adhésion à l'une ou l'autre des tenures existantes (régime seigneurial aux Français, franc et commun soccage aux Anglais) et les motivations qui sous-tendent cette adhésion; 2) l'aversion supposément éprouvée par la bourgeoisie marchande à l'endroit de la tenure seigneuriale. Fernand Ouellet, entre autres, fidèle aux postulats de l'approche psycho-culturelle qui guide son oeuvre, soutient que les marchands anglophones sont intéressés par la propriété seigneuriale pour des raisons d'ordre économique (exploitation forestière, production et commercialisation du blé) alors que les francophones répondent à leur penchant naturel pour les valeurs aristocratiques ${ }^{6}$. Il affirme au sur-

${ }^{6} \quad$ Fernand Ouellet, «Propriété seigneuriale et groupes sociaux dans la vallée du 212 . 
plus que malgré une nette tendance à l'appropriation seigneuriale, la bourgeoisie marchande «fait naître par son activité des tensions dans l'institution seigneuriale, entre en conflit avec les nobles et dans un dernier temps, remet en question le système féodal»? ${ }^{7}$.

\section{1) Dimension ethnique et appropriation foncière}

Notons en premier lieu qu'une appréciation culturaliste de l'appropriation foncière s'accorde mal aux faits. Il semble bien que, dans l'ensemble, l'attachement des francophones à la tenure seigneuriale ne soit pas aussi exclusif qu'on le laisse souvent entendre. Ainsi, un survol rapide des journaux et appendices de la Chambre d'Assemblée du Bas-Canada durant les années 1830 nous apprend que Gustave Joly, seigneur de Lotbinière, demandait en 1835 que soit changée la tenure de la partie non concédée de sa seigneurie ${ }^{8}$. Des propriétaires terriens aussi francophones que Louis Lacroix, J.-B. Giroux et Elzéar Bédard demandaient au cours des années 1831-32 que leur propriété soit désormais régie par la tenure en franc et commun soccage ${ }^{9}$. De même, on note que B. Joliette acceptait en 1835 au nom des héritiers de l'honorable Charles Gaspard de Lanaudière (son beau-père) 2010 acres concédées en franc et commun soccage dans l'arrière de la seigneurie de Lavaltrie ${ }^{10}$. Enfin, que des individus avec des noms à consonance aussi aristocratique que Pierre de Boucherville, $\mathrm{H}$. de Rouville, De Salaberry, Pascal de Sales Laterrière, P.-M. de La Valtrie, C. de Lotbinière, Saveuse de Beaujeu, De Montenach (sans compter des noms plus «bourgeois» tels que Fleury Deschambault, Francis Languedoc et Denis Létourneau) apparaissent sur la liste des personnes à qui furent concédées des terres en franc et commun soccage dans les Cantons de l'Est ${ }^{11}$, révèle à tout le moins une loyauté douteuse à l'égard du système seigneurial et des valeurs aristocratiques qui s'y rattachent.

Il est assez difficile de comprendre pourquoi l'attitude des francophones et des anglophones en matière foncière aurait répondu à deux types de logique différents. Suivant le raisonnement culturaliste, il faudrait admettre que les motifs qui incitaient les Canadiens français à faire commuer la tenure de leur propriété foncière ou à accepter des concessions régies par le franc et commun soccage auraient différé des motifs supposément capitalistes

\footnotetext{
Ibid, 204.

8 Journal de la Chambre d'Assemblée du Bas-Canada, (JCABC), 20 novembre $1835,175$.

${ }_{9}$ Ibid, 1833, appendice KK.

10 Ibid, 1835-1836, appendice CC.

"Ibid, 1833, appendice NN.
} 
qui amenèrent un Edward Ellice, seigneur anglophone de Beauharnois, à demander une commutation de tenure sur une partie de sa seigneurie. À notre avis, rien n'autorise une pareille conclusion. De même, rien ne permet de présumer non plus que le comportement économique d'individus tels que J. Masson, seigneur de Terrebonne et B. Joliette, seigneur de Lavaltrie, ait été conditionné d'abord par des considérations de statut social; leurs activités économiques caractérisent plutôt deux hommes d'affaires avant tout, manifestement préoccupés par la réalisation de profits.

Notons enfin que les sources consultées ne révèlent pas davantage un mouvement populaire en faveur de l'expansion du territoire seigneurial au moment où les seigneuries existantes deviennent surpeuplées en raison des pressions démographiques. Les revendications populaires vont plutôt dans le sens d'une meilleure accessibilité à la terre; on dénonce vigoureusement les excès et les abus des propriétaires fonciers, quel que soit le mode de tenure auquel ils sont rattachés ${ }^{12}$.

Certes, les Canadiens français, notamment par le discours patriote, semblent peut-être dénoncer plus sévèrement le système de franc et commun soccage que la tenure seigneuriale. Cela peut se comprendre. En effet, dans le contexte de l'époque, privilégier le système seigneurial constitue un moindre mal; les terres sont concédées et non vendues. Mais les dénonciations patriotes du franc et commun soccage ne se présentent pas comme une sacralisation du système seigneurial, bien au contraire. À la veille des insurrections, sous l'effet des pressions émanant de la base - on peut en prendre la mesure en consultant les résolutions adoptées dans les assemblées de comté - le discours patriote se fait plus virulent à l'égard de cette tenure. Plusieurs insurgés croiront même, en s'engageant, lutter entre autres pour son abolition ${ }^{13}$.

Devant ces faits, il serait exagéré de soutenir que les francophones ne juraient que par le système seigneurial et ses valeurs féodalistes. L'adhésion de certains à un mode de tenure qui, dans son principe contrevenait à la tenure seigneuriale, incite à nuancer l'appréciation que l'on fait de toute cette question. Il ne s'agit pas de nier que les valeurs nobiliaires inhérentes au système seigneurial aient pu exercer un attrait quelconque sur certains propriétaires francophones de seigneuries. Il s'agit plutôt d'éviter les jugements

12 Voir entre autres pétitions concernant le régime seigneurial: la pétition des habitants de la seigneurie de Beauharnois, JCABC, 15 décembre 1832; la pétition de censitaires de Noyan, Foucault et Lacolle, JCABC, ler décembre 1831; la pétition des habitants, censitaires et propriétaires de Lotbinière, JCABC, 24 janvier 1834.

13 Voir le «Rapport des Commissaires sur la tenure seigneuriale», Journal de l'Assemblée législative de la Province du Canada, 1843, témoignage de F.B. Ricard. 
trop généraux. Cet attrait n'est pas partagé par l'ensemble du groupe francophone, non plus qu'il constitue une motivation qui lui soit exclusive.

En outre, à l'heure où la propriété foncière constitue encore l'assise du pouvoir politique en Angleterre, plusieurs anglophones s'intéressent ici à l'acquisition de seigneuries. Durant les premières décennies qui suivent la Conquête, certains d'entre eux se voient octroyer des seigneuries abandonnées par leurs titulaires ${ }^{14}$. Dans les décennies subséquentes, d'autres anglophones feront l'achat de seigneuries mises en vente ${ }^{15}$. Au surplus, il existe des cas d'anglophones qui, possédant déjà des terres en franc et commun soccage, en demandèrent la commutation sous tenure seigneuriale. Ainsi en est-il de James McCallum qui constitua les seigneuries de SaintJacques et de Saint-Normand et de Jacob Mountain qui fit créer la seigneurie de Thwaite, tous deux à partir de terres détenues précédemment en franc et commun soccage ${ }^{16}$.

De même, tous les anglophones n'adhèrent pas sans réserve aux principes de la tenure soccagère et de l'organisation socioéconomique qui s'y rattache. Il n'est pas peu révélateur par exemple qu'un représentant anglais du comté de Stanstead à la Chambre d'Assemblée, E. Peck, s'oppose en 1831 à l'imposition des lois anglaises dans les cantons selon la loi des Tenures de 1825, arguant en substance que celles-ci ne sont pas nécessairement bien adaptées aux conditions du pays. Lors d'une intervention en Chambre, il affirme que les lois de primogénitures dérivées du code anglais posent dans un nouveau pays certaines difficultés quant aux transferts de propriété. Aussi les lois anglaises lui semblent incompatibles avec les habitudes de vie que les gens ont développées ici. Il mentionne, par surcroît, que ces lois «leur avaient été imposées et cela contrairement à leurs désirs et à leurs intérêts». Il conclut que ces lois devaient être rappelées et remplacées par les lois françaises qui, malgré leurs vices, sont encore préférables aux lois anglaises ${ }^{17}$.

À la lumière de ces divers éléments, il est aisé de voir ce qu'a de spécieux une appréciation de la propriété foncière au BasCanada selon un découpage ethnique et/ou culturaliste. Il n'y a pas, proprement dit, de racialisation du système foncier bascanadien; on ne se prononce pas, simplement en vertu de son origine ethnique, pour ou contre un mode particulier de tenure. 1978), 24

4 S.B. Ryerson, Le capitalisme et la Confédération (Montréal, Partis-Pris,

15 Fernand Ouellet, op. cit., 196-204.

16 Georges Baillargeon, Influence du conflit seigneur-censitaire sur la politique canadienne, mémoire de maîtrise (science politique) (Université de Montréal, 1976), 152.

17 J.P. Bédard, Histoire de Cinquante ans (1791-1841) (Québec, Léger Brousseau, 1869), 306. Voir aussi Rapport des débats, La Minerve, 17 mars 1831. 


\section{2) Bourgeoisie marchande et tenure seigneuriale}

La prétendue opposition de la bourgeoisie marchande aux structures féodalistes de la formation sociale est tout aussi problématique. Malgré certaines demandes de commutation de tenure faites par des seigneurs marchands, il serait faux de croire qu'il existait avant les années 1840 au sein de la bourgeoisie marchande un sentiment généralisé d'opposition à la tenure seigneuriale bascanadienne. Le fait qu'une proportion fort considérable de bourgeois autant anglophones que francophones se soit portée acquéreurs de seigneuries entre 1782 et 1840 indique, sinon un engouement, à tout le moins une très grande tolérance à l'égard du système seigneurial de la part de la bourgeoisie en général. On estime que 53,9\% des acheteurs de seigneuries au cours de cette période appartiennent à la bourgeoisie anglophone; ils font l'achat de $52,7 \%$ des seigneuries mises en vente. Les bourgeois francophones sont eux aussi très actifs. Ils constituent $36,7 \%$ du nombre total d'acheteurs de seigneuries et se procurent $38 \%$ des seigneuries mises en vente. Au total, la bourgeoisie tant anglophone que francophone s'appropriera $90,7 \%$ des seigneuries disponibles sur le marché immobilier et constituera une proportion de $90,6 \%$ de tous les acheteurs ${ }^{18}$.

Somme toute, au cours de la période considérée, l'opposition de la classe marchande au système seigneurial sera plutôt pusillanime. Entre 1826 et 1836, 21 requêtes de commutation seront présentées au Conseil Exécutif en vertu de l'Acte des tenures du Canada (1825) autorisant l'affranchissement des droits seigneuriaux. De ce nombre, 5 seulement ont trait aux terres nonconcédées ou encore à une partie des terres déjà concédées, mais en aucun cas à la seigneurie dans son entier ${ }^{19}$. Le reste des demandes porte sur des terrains ou lots de terres possédés dans la région urbaine de Québec. Dans ces derniers cas, le coût généralement très élevé des lods et ventes dans les zones urbaines ${ }^{20}$ expliquerait

18 Fernand Ouellet, «Propriété...», 203. Il faut toutefois observer que Ouellet regroupe indistinctement sous l'épithète de bourgeois les fonctionnaires, les professionnels et les marchands, sans indiquer la proportion dans laquelle chacune de ces catégories participe à l'achat de seigneuries. Néanmoins, les exemples auxquels il fait référence permettent de penser que les marchands devaient être les plus actifs dans le domaine de l'appropriation foncière seigneuriale. Ainsi, Ouellet rapporte ailleurs que les seuls marchands anglais exploitaient en 1831, 84 seigneuries, 35 portions de seigneuries et 35 arrière-fiefs. (Éléments d'histoire sociale du Bas-Canada (Montréal, Hurtubise HMH, 1972), 100).

$19 \mathrm{JCABC}, 1833$, appendice KK et 25 novembre 1835 .

20 Redevance payable au seigneur par le censitaire suite à la vente de sa terre ou à des améliorations physiques, les lods et ventes étaient nécessairement plus élevés dans les villes en raison de changements plus fréquents de propriétaires et du renchérissement de la valeur foncière attribuable au nombre et à la valeur plus considérable des améliorations à la propriété. Dans les villes, la tenure seigneuriale constituait d'emblée un obstacle onéreux à l'établissement de quelque entreprise. 
en bonne partie les demandes de commutation; quoi qu'il en soit, il ne semble pas s'agir d'un phénomène généralisé dans les villes, là même où les commutations auraient été des plus avantageuses.

Enfin, il est intéressant de signaler qu'un certain nombre d'anglophones feront la demande de terres ou terrains régis par la tenure seigneuriale alors même qu'ils auraient pu se prévaloir des avantages de la tenure en franc et commun soccage. Parmi eux notons les noms de Munro et Bell, marchands de Trois-Rivières ${ }^{21}$.

En réalité, après la promulgation de l'acte des Tenures du Canada, on observe une indifférence quasi totale face à la possibilité qui se présentait dès lors aux seigneurs d'affranchir leurs propriétés. Même le fameux cas Ellice, que l'historiographie libérale présente comme un exemple d'opposition capitaliste à la tenure seigneuriale ${ }^{22}$, doit être abordé avec une extrême réserve.

Edward Ellice, seigneur de Beauharnois, enrichi grâce au commerce des fourrures, très actif ici et en Angleterre dans les milieux économique et politique, voulut une première fois, en 1822 , affranchir sa seigneurie des droits féodaux. Dissuadé par son agent canadien d'exécuter son projet, il fit tout de même pression auprès du Parlement impérial qui promulgua l'Acte des Tenures en 1825 . Il revint à la charge en 1831 et obtint que la tenure régissant la partie non concédée de sa seigneurie soit commuée.

Bien qu'affichant une apparente rhétorique capitaliste ${ }^{23}$, Ellice est mû en réalité par des intérêts et un esprit d'agioteur et de mercantiliste. Entre 1810 et 1821 il refuse, par l'intermédiaire de son agent, de procéder à quelque concession que ce soit afin de faire hausser le prix des terres sur sa seigneurie. Lors de sa première demande de commutation en 1822, Ellice, propriétaire absentéiste, cherche en fait à obtenir la "propriété absolue» de ses

21 JCABC, 1833, appendice KK.

22 Fernand Ouellet insiste particulièrement sur le cas d'Edward Ellice comme preuve de la volonté de certains marchands anglo-saxons d'abolir le régime seigneurial au profit du développement du capitalisme: «En 1833, écrit-il, (Ellice) déclare ouvertement son hostilité aux servitudes permanentes et affirme sa croyance dans le caractère absolu de la propriété... Non seulement Ellice manifeste sa préférence pour le franc et commun soccage mais il développe sa seigneurie dans un esprit capitaliste.» (Fernand Ouellet, Le BasCanada 1791-1840. Changements structuraux et crise (Ottawa, Éditions de l'Université d'Ottawa, 1976), 233).

${ }_{23}$ En 1828, Ellice déclare: «Le principal obstacle à l'amélioration du Bas-Canada vient des objections qu'ont les sujets britanniques à appliquer sur des biens-fonds les profits considérables qu'ils ont retirés du commerce du pays, et des empêchemens opposés à la circulation du capital ainsi appliqué par les dispositions de la tenure féodale et les primes considérables exigées à chaque aliénation... Je puis ajouter que la valeur des propriétés dans les villes a éprouvé depuis quelques années une baisse formidable, et je ne puis rien voir dans l'état présent du Canada, qui promette un état de choses différent, excepté par l'éloignement des obstacles actuels à l'application des capitaux à l'amélioration du pays.» F. Ouellet, Histoire économique et sociale du Québec, 1760-1850 (2 tomes, Montréal, Fides, 1971), II: 353 . 
terres non concédées; il lui semble plus avantageux de vendre les dites terres que de les concéder. Suite aux pressions de son agent qui lui fait comprendre qu'en raison de la pauvreté relative de la population il serait illusoire d'espérer tirer rapidement profit de la vente de ses terres, il abandonnera son projet. Ces diverses tractations donnent à penser qu'Ellice est vraisemblablement plus préoccupé par de probables gains de spéculation foncière que par la liberté d'établissement et de développement que lui aurait permis une commutation.

En 1831, par sa deuxième requête de commutation, le seigneur de Beauharnois n'espère plus seulement vendre ses terres non concédées à bon prix. Il cherche maintenant à profiter d'une erreur d'arpentage en sa faveur qui lui donnerait 32503 arpents de plus que ne l'autorise la concession originale. Or, deux cents familles vivent sur ces terres. Avec l'erreur d'arpentage, elles se retrouvent sans titre foncier et donc obligées envers Ellice qui, par la commutation, parvient à se faire reconnaitre un droit de propriété sur les 32503 arpents. En outre, grâce à une évaluation foncière truquée ${ }^{24}$, il n'aura à payer qu'une indemnité minime pour les droits de commutation. Il acquiert ainsi en franc et commun soccage la propriété des 118000 acres de terres non concédées de sa seigneurie. Dans ces conditions, il devient aisé de comprendre pourquoi le seigneur de Beauharnois se fait un ardent défenseur de la tenure en franc et commun soccage.

Cependant, les motifs qui poussent ce seigneur à faire commuer la tenure d'une partie de sa seigneurie ne ressemblent pas, à notre avis, à une profession de foi capitaliste (au sens du développement de la sphère de production), mais répondent essentiellement à la logique mercantiliste. L'accumulation foncière ne représente pas ici un moment de l'investissement de capital productif, mais constitue plutôt un objet d'échange et de spéculation. En cela, elle reste soumise aux impératifs de la sphère de circulation de la structure économique.

L'Acte des Tenures du Canada qui, dans l'esprit de ses formulateurs, devait conduire à l'abolition du système seigneurial au Bas-Canada, ne servira à toutes fins utiles que d'instrument de spéculation aux mains des seigneurs désireux d'obtenir la propriété absolue de leurs terres encore non concédées. "Ces derniers, explique Baillargeon, détournèrent totalement cette loi de son but, en

24 Les évaluateurs déclarèrent sans valeur 23000 acres de terres dont certaines se révéleront plus tard comme les plus fertiles de la seigneurie. Les 95000 autres acres de terres non concédées furent évaluées à 0.60 cents l'acre alors que les terres de la Couronne se vendaient 0.75 cents l'acre et qu'Ellice vendait ces mêmes terres à $\$ 6.00 /$ acre. Le droit de commutation n'étant que d'un vingtième, Ellice ne paya que 0.03 cents l'acre les 95000 acres retenues par les évaluateurs. (Tiré de G. Baillargeon, op. cit., 149). 
commuant les droits seigneuriaux de la Couronne non pas sur toute leur seigneurie, mais exclusivement sur l'augmentation non peuplée de cette dernière. ${ }^{25}$ En affranchissant de droits féodaux la totalité du domaine seigneurial, les seigneurs devaient s'attendre à ce que, en toute logique, leurs censitaires se prévalent de cet affranchissement. Ce faisant, ces derniers se libéreraient de la tutelle et, surtout, des redevances seigneuriales, privant du même coup le seigneur de revenus garantis. C'est ce qui explique pourquoi les quelques seigneurs qui tirèrent profit du droit de commutation ne le firent que sur les parties non concédées de leur seigneurie, n'ayant aucunement comme objectif l'abolition totale du système seigneurial, qui aurait impliqué la renonciation aux revenus découlant des droits seigneuriaux ${ }^{26}$. En se limitant à la commutation sur la partie non-concédée, les seigneurs se voyaient ainsi affranchis des prescriptions du droit seigneurial, notamment du devoir de concéder sur demande, et pouvaient dès lors espérer tirer plus grand profit de la vente de ces terres (spéculation et libre exploitation du potentiel forestier).

À la lumière de ces précisions, nous croyons être en mesure d'affirmer que, dans le contexte structurel du Bas-Canada d'avant 1846, les propriétaires de seigneuries, quelle que fût leur assise sociale ou leur origine ethnique, n'avaient pas d'intérêt immédiat à vouloir l'abolition du régime seigneurial. En soi, les éléments structurels de type féodal de la formation sociale n'étaient pas en contradiction avec les activités mercantilistes auxquelles s'adonnaient plus de la moitié des propriétaires de seigneurie.

\section{II - Le paradoxe mercantiliste: mercantilisme et reproduction de rapports de production de type féodal}

Nous ne nions pas l'existence au cours de cette période d'une accentuation des tensions menant éventuellement à l'abolition/éclatement du régime seigneurial et à laquelle la bourgeoisie marchande n'est pas totalement étrangère ${ }^{27}$, pas plus que la lente

25 Ibid., 158. Ainsi Ellice, neuf ans après avoir obtenu l'affranchissement des droits seigneuriaux sur une partie de la seigneurie, n'avait pas encore eu la magnanimité d'étendre les mêmes avantages aux censitaires établis sur la partie concédée.

${ }_{26}$ «Rapport des Commissaires sur la tenure seigneuriale», Journal de l'Assemblée Législative de la Province du Canada, 1843, appendice F. Témoignage de John McDonald, Robert Brodie et Donald Finlayson.

27 La répulsion que disent éprouver les marchands à l'égard du système seigneurial n'est que rhétorique tant et aussi longtemps que les rapports d'exploitation seigneuriaux leur permettent d'assurer leur survie en tant qu'agents du capital marchand. Or c'est précisément le cas jusqu'au milieu des années 1840 . Ce n'est qu'après le déclin de l'économie marchande amorcé durant ces mêmes années que, dans plusieurs milieux, on réclamera de manière plus insistante que jamais l'abolition du système seigneurial en raison de l'entrave qu'il constitue alors à la libre circulation du capital, à l'investissement dans la sphère de production, à la commercialisation de la production agricole et à la création d'un marché national. 
émergence d'une sphère de production et de rapports de production capitalistes encore dominés, par suite de la mise en valeur du bois après 1807. Cependant, notre objectif immédiat est de mettre en lumière le caractère non capitaliste des rapports de production dominants et de démontrer que, malgré une tendance à la rationalisation capitaliste manifestée par un certain nombre de seigneurs dans l'administration de leur propriété, les structures seigneuriales et le maintien des rapports de production afférents sont nécessaires à la reproduction de l'économie marchande. De même, on verra que les pratiques d'exploitation foncière sur le territoire non seigneurial (tenure soccagère) produisent dans bien des cas des effets similaires.

Cette perspective d'analyse suggère deux choses. D'une part, la validité des interprétations qui associent la bourgeoisie marchande à une classe capitaliste au sens propre est mise en cause. D'autre part, cette dominance de rapports de production noncapitalistes, de même que leur capacité de se reproduire (même imparfaitement) au sein de l'économie marchande, permet d'entrevoir un mouvement de résistance à la pénétration généralisée du capitalisme dans la formation sociale. Cette résistance ne saurait être que temporaire car, ainsi que nous le verrons plus loin, les pratiques foncières liées à l'économie marchande vont quand même permettre la mise en place de certains aspects apparentés à la réalité capitaliste. En effet, elles seront largement responsables du processus de dépossession/prolétarisation des masses paysannes. De même l'économie marchande du bois donnera naissance à un secteur de petite production annonciateur de rapports, de production et de structures, capitalistes.

\section{1) Régime seigneurial et pratiques foncières}

La fonction colonisatrice du régime seigneurial s'altéra considérablement au cours des années. Fondamentalement, «la seigneurie canadienne n'était pas vraiment différente de sa contrepartie française... (et) reposait en gros sur le même enchevêtrement de droits féodaux et de droits de caractère seigneurial» ${ }^{28}$. Le censitaire ne possédait que des droits de propriété fort limités, ce qui laissait au seigneur la propriété réelle et presque absolue des moyens de production. Après 1800 , le caractère d'exploitation féodale du système seigneurial devient de plus en plus marqué ${ }^{29}$.

Ainsi plusieurs seigneurs faillirent à leur rôle supposé «d'agent de colonisation» en se livrant à des pratiques qui s'éloi-

${ }^{28}$ F. Ouellet, "Libéré ou exploité! Le paysan québécois d'avant 1850», Histoire sociale/Social History, 13, 26 (novembre 1980): 345-346.

29 Ibid.: 348. 
gnaient considérablement des objectifs originaux de la tenure seigneuriale et des prescriptions du droit coutumier français qui la régissait. Une pétition présentée par divers censitaires à la Chambre d'Assemblée le 16 mars 1825 fait clairement état de ces pratiques. Les pétitionnaires remarquent en effet

qu'une portion considérable de terres de cette Province, tenues en fief et seigneurie, est depuis long-tems sujète à une administration vicieuse, à la fois dommageable aux intérêts de l'agriculture et du commerce de ce pays, et tout-à-fait contraire aux intentions sages et bienveillantes qu'avoit le Gouvernement en faisant ces octrois. Une étendue considérable de ces terres est encore en ce moment dans son état de nature, au grand détriment du pays, dont l'établissement se trouve par-là grandement retardé et les Pétitionnaires prennent la liberté de faire observer que ces terres ne demeurent pas incultes par l'impossibilité de les faire établir, comme on pourroit raisonnablement l'inférer d'après le grand nombre d'émigrans qui arrivent tous les ans dans cette Province, et d'après la densité de la population agricole du pays; mais ou parce que les présens propriétaires ne veulent pas concéder ces terres, désirant tirer avantage de l'augmentation de la valeur des propriétés foncières, afin de pouvoir en retirer par la suite de plus fortes rentes et autres droits lucratifs, faisant par-là dégénérer la munificence royale en une spéculation sur les terres; ou à cause de l'administration des seigneuries, ou de leur tenure, qui a fait naître et a accrédité dans la Grande-Bretagne et l'Irlande des préjugés défavorables au Bas-Canada; ce qui, à moins qu'il soit adopté des mesures sages pour faire disparô̂tre la cause de ces préjugés, continuera à nous priver des avantages de l'émigration, tandis que nos voisins et la province supérieure voient augmenter tous les ans leur population et leurs richesses par l'accession d'un grand nombre de cultivateurs industrieux. ${ }^{30}$

Sept «inconvénients» attribuables aux pratiques seigneuriales sont ensuite énumérés. On y déplore: 1 . le fait que les seigneurs se réservent de grandes étendues de terres en bois debout sans en concéder une seule parcelle ou, le cas échéant, d'en offrir les parties de médiocre qualité; 2 . que pour se soustraire à leur obligation de concéder sur demande les terres non cultivées de leur seigneurie, certains seigneurs s'assurent la complicité de personnes fiables à qui ils concèdent de vastes étendues de terres pour que celles-ci les vendent ensuite en faisant participer le seigneur au profit de la vente; 3. que, par négligence, certains seigneurs concèdent plus d'une fois le même lot à différentes personnes obligeant l'occupant du moment à quitter les lieux pour faire place au nouveau; 
4. qu'avant de les concéder, certains seigneurs dépouillent leurs terres du meilleur bois de construction afin de le vendre, diminuant ainsi la valeur de la terre et empiétant sur les droits de la Couronne (le chêne et le pin devaient être réservés à la fabrication des vaisseaux de Sa Majesté); 5. l'imprécision dans la rédaction des contrats de concession, qui permet par la suite au seigneur d'imposer au censitaire de nouvelles obligations: transport des cens et rentes au lieu fixé par le seigneur, peu importe la distance à parcourir; arpentage à ses propres frais du lot qu'il cultive aussi souvent qu'il plaît au seigneur; interdiction de vendre toute partie du bois de construction qui se trouve sur sa terre, l'empêchant même d'en faire du bois de corde et le contraignant à détruire le bois de construction abattu lors des défrichements aux fins de culture; 6 . la négligence de certains seigneurs à entretenir des moulins banaux propres à la fabrication de farine exportable obligeant le cultivateur à offrir son grain à perte au marché faute d'avoir pu le raffiner et le moudre; la négligence de bâtir des moulins dans les nouveaux établissements forçant les cultivateurs à parcourir de longues distances pour faire moudre leur grain; 7. que l'augmentation rapide des cens et rentes couvre le cultivateur de redevances onéreuses que le nouveau colon ou l'émigrant sont souvent incapables d'acquitter; en conséquence on affirme que le seigneur devrait se contenter de percevoir seulement les lods et ventes étant donnée l'augmentation graduelle de revenus que l'amélioration des terres lui assure.

Ces doléances particulières ne sont pas simplement le fait isolé de quelques cultivateurs indûment exploités; les journaux et appendices de la Chambre d'Assemblée comptent bon nombre de pétitions semblables, dont certaines se font carrément revendicatrices et remettent parfois en cause les principes mêmes de la propriété seigneuriale ${ }^{31}$.

Au cours de la période considérée, les seigneurs accentuent la nature féodale des rapports de production qui régissent le système seigneurial pour finalement accaparer les sources d'énergie et autres moyens de production rattachés à la propriété foncière. Se fondant sur leur seul droit de banalité, lequel autorise le seigneur à

31 Voir par exemple la pétition des habitants de Russeltown (seigneurie de Beauharnois), JCABC, 15 décembre 1832; la pétition des censitaires de Noyan, Foucault et Lacolle, JCABC, 12 décembre 1831; la pétition des habitants, censitaires et propriétaires de la Seigneurie de Lotbinière, JCABC, 25 janvier 1834; la pétition des habitants de la Seigneurie De Léry en faveur d'une réglementation à propos du prélèvement des cens et rentes, JCABC, 4 mars 1835; la pétition des habitants de la Seigneurie de Lacolle contre les abus du seigneur, JCABC, 13 novernbre 1835 . Voir aussi les témoignages contenus dans certains rapports de comités parlementaires sur la question des terres: Appendice NN, JCABC, 1832-1833; Appendice EEE, JCABC, 1835-1836. 
exiger des censitaires qu'ils utilisent le moulin seigneurial pour moudre leur grain destiné à la consommation domestique, bon nombre de seigneurs en vinrent à contraindre leurs censitaires à moudre au moulin seigneurial tout leur grain, y compris celui destiné à la commercialisation. Ils s'arrogèrent aussi le droit de prendre, d'inonder et de diviser sans qu'aucune compensation ne soit offerte aux censitaires, toute portion de terre qui sur le domaine seigneurial, pouvait servir à l'installation, à l'approvisionnement en eau et au fonctionnement du moulin banal.

Certains seigneurs s'approprièrent aussi tout le bois de sciage et une partie du bois de chauffage qui se trouvaient sur les terres de leurs censitaires. D'autres se réservèrent encore les richesses naturelles qui pouvaient se trouver à la surface du sol ou dans le soussol. Enfin, là où la chasse et la pêche constituaient des activités commerciales, les seigneurs se ménagèrent un droit d'imposition sur le produit de la chasse et/ou de la pêche réalisé par les censitaires $^{32}$.

La plupart des seigneurs ne manquèrent pas en outre de se prévaloir du droit de corvées obligatoires qui leur permettait de requérir la participation non rémunérée de leurs censitaires à la réalisation de travaux d'amélioration générale du domaine seigneurial (construction de chemin, réfection du moulin, érection d'une église, coupe de bois, etc.).

Notons finalement que pour contourner la fixité des redevances (cens et rentes) prescrites par le droit français en ce qui touche les terres déjà concédées, plusieurs seigneurs eurent recours au titre nouvel. Il s'agit d'une pratique légale qui oblige un censitaire endetté à prendre un nouveau titre de propriété aux conditions fixées par le seigneur. Par cet acte notarié, le paysan s'engage à payer les arrérages dus sur sa terre et à payer le nouveau taux de rentes fixé par le seigneur ${ }^{33}$. Dans les faits, cette pratique permettait au seigneur d'ajuster les taux de rentes dans les anciennes concessions à ceux en vigueur dans les nouvelles.

S'il est vrai que toutes ces pratiques assurent aux seigneurs une certaine accumulation de capital tout en ayant pour effet d'engendrer certaines conditions propices au développement du capitalisme, notamment la dépossession de la paysannerie qui résulte des charges onéreuses et de la non accessibilité à la terre, cela ne saurait nous autoriser à parler de seigneuries «capitalistes» dans le

32 Toutes ces informations sur l'accaparement des droits seigneuriaux proviennent de Baillargeon, op. cit., 52-55.

33 Lise Pilon-Lê, "Le régime seigneurial au Québec: contribution à une analyse de la transition au capitalisme», Les Cahiers du socialisme, 6 (automne 1980): 165. 
Bas-Canada d'avant l'Union. Car la volonté d'accroître leur participation au commerce du bois et à la mise en valeur de ses dérivés, conduit certains seigneurs à renforcer le caractère féodal de l'exploitation des censitaires.

Dans sa thèse sur l'endettement paysan au Québec, Lise Pilon-Lê relève ce phénomène ${ }^{34}$. Ainsi, par exemple, elle nous dit que Ellice, seigneur de Beauharnois, limite à dessein l'établissement des colons dans le but de faire monter la valeur des terres et des rentes. Nous avons vu précédemment qu'en 1831 il rachète les droits seigneuriaux de la Couronne, convertit la tenure des terres non concédées de sa seigneurie en franc et commun soccage et obtient ainsi la propriété capitaliste de ces terres-là. À la même époque, en plus de saisir les terres auxquelles on avait négligé de donner un titre foncier, il augmente la rente à plus de 9 sols l'arpent et il poursuit en justice les censitaires endettés envers lui. De sorte que, entre 1823 et 1841 , le revenu net de la seigneurie de Beauharnois allait être multiplié par 19. Cette hausse considérable de profit est attribuable à l'augmentation des cens et rentes ainsi que des lods et ventes, ces deux droits constituant $80 \%$ du revenu seigneurial total. Malgré une conjoncture agricole et économique plutôt défavorable, on parvient, dans certaines paroisses de la seigneurie de Beauharnois, à atteindre des rendements agricoles qui, certaines années, doublent presque la production de quelques-unes des meilleures seigneuries de la province. Plus tard, au cours des années 1840, Ellice s'associe à des financiers et spéculateurs britanniques avec lesquels il financera la construction du canal de Beauharnois. Les bénéfices réalisés grâce à la mise sur pied de ce projet et à la gestion de sa seigneurie seront réinvestis en Angleterre, aux États-Unis et au Haut-Canada.

L'exemple de la seigneurie de Lavaltrie dirigée par B. Joliette, est aussi fort significatif ${ }^{35}$. De 1822 à 1832 , au moment où le commerce du bois est en plein essor, le seigneur met en place une politique pour augmenter le revenu seigneurial et l'investir dans la construction et l'exploitation de moulins à scie. Ainsi, en deux ans, de 1823 à 1825 , il parvient à récupérer $93,4 \% 0^{36}$ de la somme totale des arrérages qui lui étaient dus par ses censitaires, soit la somme de 74555 livres tournois. Les censitaires acquittent leur dette en bois et en travail pour le seigneur. Profitant de la conjoncture favora-

\footnotetext{
34 'Lise Pilon-Lê, L'endettement des cultivateurs québécois. Une analyse socio-historique de la rente foncière: 1670-1904, thèse de doctorat (Anthropologie) (Université de Montréal, 1977), 248-258.

35 Voir J.-C. Robert «Un seigneur entrepreneur, Barthélémy Joliette et la fondation du village d'Industrie, 1822-1850», Revue d'histoire de l'Amérique française, 26, 3 (décembre 1972): 375-397.

36 Pourcentage calcuié à partir des chiffres fournis, ibid, 384.
} 
ble au commerce du bois, le seigneur de Lavaltrie exploite les boisés (terres non concédées) de sa seigneurie. Cette activité nécessite le remembrement du domaine seigneurial pour y ériger un moulin à scie. Pour ce faire, le seigneur utilise son droit de retrait censuel contre les censitaires endettés envers lui; il confisque leur concession pour la rattacher au domaine seigneurial. En 1823, un moulin à scie est construit au coût de 17300 livres tournois. Le seigneur y transforme ce qu'il exploite sur son domaine en bois de sciage. Parallèlement, il entreprend une série de poursuites judiciaires contre ses censitaires pour recouvrer ses arrérages et les obliger à moudre leur blé au moulin banal. Les jugements qui font suite aux procès lui sont toujours favorables et lui permettent de retirer aux paysans leur droit d'usage sur leurs terres et de saisir le bois qu'ils ont coupé. De plus, dans ces procès, il fait payer aux censitaires les jugements de Cour et les frais de saisie. Enfin, il exige le paiement des lods et ventes lors de donation de terre entre père et fils. Ces divers procédés, ajoutés au paiement courant des cens et rentes, permettent au seigneur de Lavaltrie de réaliser des profits considérables entre 1822 et 1844 . Durant cette période ses revenus atteignent 757555 livres tournois et ses dépenses sont de l'ordre de 181972 livres tournois ${ }^{37}$. Le montant moyen de la rente par censitaire triple en huit ans. Dans un tel contexte, il va sans dire que les dépenses d'investissement sont rapidement amorties; au cours de la seule année 1825, le seigneur vend pour 37563 livres tournois de madriers, soit plus de deux fois le coût de construction du moulin à $\operatorname{scie}^{38}$.

Madame Pilon-Lê estime que ces deux exemples illustrent bien l'existence de tentatives de la part des seigneurs de capitaliser la rente seigneuriale, en poussant au maximum l'exploitation des censitaires pour investir le capital accumulé dans des activités capitalistes. Cependant, il convient de bien voir dans quel sens joue la mise en oeuvre de ces activités en apparence capitalistes. Les seigneurs qui s'adonnent à de telles pratiques manifestent en réalité leur volonté de monopoliser les moyens de production et les forces productives liés à l'échange (sphère de circulation).

Dans le cadre d'une économie à dominante marchande, la capitalisation de la rente foncière est essentiellement tributaire des impératifs du capital marchand; tant que persiste, à tout le moins, le caractère mercantiliste dominant des fonctions économiques. La construction de canaux ou l'érection de moulins à scie sont

37 J.-C. Robert, L'activité économique de Barthélémy Joliette et la fondation du village d'Industrie (Joliette) 1822-1850, thèse de M.A. (histoire) (Université de Montréal, 1971), 78 et 101 .

38 J.-C. Robert, «Un seigneur...», 387. 
d'abord motivées par la nature des activités commerciales en cours dans la colonie. Elles sont directement fonction des échanges avec la métropole; d'une part, les nouveaux canaux dans Beauharnois sont essentiels à l'amélioration des échanges marchands, et, d'autre part, le développement de l'industrie du bois dans Lavaltrie est complètement dépendant de la demande britannique pour ce produit.

En effet, le commerce du bois connaît pendant ces années une expansion sans précédent ${ }^{39}$ qui incite naturellement les seigneurs à y participer. Le cadre seigneurial, par l'assujétion économique des censitaires aux volontés du seigneur, permet effectivement une exploitation à fort bon compte des potentialités forestières du domaine seigneurial ${ }^{40}$.

Ainsi donc, bien que les initiatives économiques de certains seigneurs puissent revêtir une apparence typiquement capitaliste, elles s'accomplissent en réalité non pas sur la base d'extorsion de plus value, ce qui est le propre du capitalisme, mais sur la base de corvées obligatoires, du travail gratuit en remboursement de dettes et de rapports de soumission/exploitation de nature essentiellement féodaliste. L'accumulation de capital reste soumise pendant ces années à la reproduction d'un rapport de production non capitaliste. En outre, elle répond a priori aux exigences de l'investissement dans la sphère de circulation et de sa reproduction élargie, sans révéler encore une volonté de développement de la sphère de production.

L'accaparement des seigneuries par la bourgeoisie marchande n'a pas encore donné avant les années 1840 une orientation typiquement capitaliste au développement de la colonie. De par la nature de leurs activités au sein du système seigneurial, les marchands s'intègrent à ses structures et semblent assurer le fonctionnement et la reproduction de ses mécanismes particuliers.

Cette reproduction est d'autant plus facilitée qu'elle s'accomplit avec la sanction d'un système juridico-politique particulièrement favorable au maintien social et économique des grands propriétaires fonciers. L'appareil judiciaire est contrôlé en large partie par des membres de ce groupe et leur position de classe se fait nettement sentir dans les décisions qui ont trait aux litiges fonciers. «Dans presque toutes les disputes entre seigneurs et censitaires qui étaient portées devant les cours de justice, les censitaires étaient déboutés;... la présence sur le banc de la justice de «seigneurs por-

39 Voir F. Ouellet, Histoire économique..., chapitre XIII.

40 Voir le témoignage de Jacques Duffroi dans le «Premier rapport du Comité permanent sur les terres et droits seigneuriaux», JCABC, 1835-1836, appendice EEE. 
tant tricorne» leur enlevait tout espoir d'obtenir gain de cause contre leur seigneur en matière de droits seigneuriaux ${ }^{41}$. Il en est de même du Conseil législatif qui, composé en grande partie de seigneurs et de spéculateurs fonciers, usera fréquemment de son veto pour faire obstacle aux législations de la Chambre d'Assemblée visant à restreindre les pratiques foncières abusives ou à faciliter l'accès aux terres tant sur le territoire seigneurial que sur les terres tenues en franc et commun soccage ${ }^{42}$.

Cette situation de prépondérance de l'instance juridicopolitique n'a rien d'étonnant. C'est la nature même des rapports entre seigneur et censitaire qui est en cause. Dans le mode de production féodale, auquel s'apparente le régime seigneurial bascanadien, le surproduit n'appartient pas de lui-même au seigneur puisque le producteur (censitaire) n'a pas encore été entièrement séparé de ses moyens de production. Par conséquent, l'appropriation du surproduit par le seigneur n'est pas directement inscrite dans le procès de production lui-même. Elle doit s'appuyer sur des prescriptions juridiques (relevant de l'instance juridico-politique) qui définissent la nature des rapports économiques entre censitaire et seigneur. Bref, ce sont les instances non-économiques qui contribuent à assurer la reproduction des rapports de production. Or, dans le mode de production capitaliste, le rôle de l'instance juridico-politique est atténué étant donné la nature différente du rapport de production qui s'établit entre propriétaire et non-

41 G. Baillargeon, op. cit., 27. Voir aussi le témoignage de J. McDonald, R. Brodie et D. Finlayson in Rapport des commissaires...

42 Notons, à titre d'exemples: le rejet en 1833 du bill de la Chambre d'Assemblée visant à venir en aide aux censitaires de la Couronne dans les districts de St-Jean et St-Roch en banlieue de Québec; le rejet d'un bill qui visait à régulariser la régie des biens des Jésuites (Les Jésuites avaient fait don au gouvernement d'une partie de leurs propriétés afin de contribuer au développement de l'instruction publique. Or, l'administration de ces terres par le gouvernement colonial avait fait l'objet de plusieurs irrégularités: on rapporte qu'un officiel en possédait 26000 acres pour lesquelles il n'avait rien déboursé, plusieurs terres et fermes situées sur le domaine des Jésuites étaient louées à des prix inférieurs à leur valeur réelle à des individus proches de l'administration et des sommes d'argent dues suite à la vente de certaines portions de terre n'avaient jamais été perçues. Le bill de la Chambre d'Assemblée visait à corriger ces abus et à diviser le domaine des Jésuites en lots convenables pour favoriser l'établissement d'agriculteurs); le rejet en 1835 du bill proposant le renouvellement de la loi contre les saisies et ventes frauduleuses de terres et autres propriétés; le rejet en 1835 d'un bill pour le rappel de loi impériale sur les tenures (1825) (La loi des tenures de 1825, basée sur le droit de primogéniture, avait pour effet de favoriser la monopolisation foncière. La Chambre d'Assemblée en avait demandé le rappel à plusieurs reprises, mais en vain). D'autres lois proposées par la Chambre d'Assemblée et qui avaient pour but de faciliter l'accès aux terres et à encourager la colonisation étaient aussi souvent déboutées au Conseil. Ainsi, en fut-il du bill pour la réparation des chemins et des ponts présenté en 1835 et qui aurait permis l'intervention directe des habitants dans l'administration des routes et des affaires locales. Le projet de loi aurait déchargé les agriculteurs de taxes inutiles et voulait aussi promouvoir le développement agricole et l'ouverture de nouveaux établissements. (Ces informations proviennent du Vindicator, 31 mars 1835, ler mai 1835, et de divers numéros du Vindicator et de la Minerve, durant avril et mai 1836). 
propriétaire des moyens de production. Dans ce cas, le producteur est totalement dépossédé de ses moyens de production. Sa soumission au capitaliste s'inscrit donc directement dans le procès de production lui-même ${ }^{43}$.

En regard des éléments présentés jusqu'ici, il semble permis d'affirmer que la bourgeoisie marchande, de même que le type d'économie sur lequel elle s'appuie s'assimilent parfaitement avant 1846 à l'environnement structurel de nature féodale qui prédomine alors au sein de la formation sociale canadienne.

Un examen plus approfondi de l'appareil superstructurel, notamment de la place de l'église, du contenu de l'idéologie dominante et de l'organisation de la structure juridico-politique permettrait d'appuyer fermement cette dernière affirmation. Malheureusement, les contraintes d'espace empêchent ici d'aller plus avant dans cette voie.

\section{2) Spéculation, monopoles fonciers et tenure soccagère}

En 1791, on le sait, le Parlement impérial instaure au BasCanada un système foncier connu sous le nom de franc et commun soccage (tenure soccagère). Ce mode de tenure libre devait, en théorie, remplacer éventuellement la tenure seigneuriale; en réalité il se développera parallèlement. On nous le présente habituellement comme une émanation du libéralisme et des principes capitalistes d'organisation de la propriété foncière; en vertu de ce système, le paysan achète sa propre terre, la gère et la développe comme il l'entend et n'est redevable à aucun seigneur. Au BasCanada, on imposera le franc et commun soccage sur la presque totalité du territoire non encore concédé à des seigneurs en 1791.

$\mathrm{Si}$, dans son principe, la tenure soccagère devait offrir une plus grande facilité d'acquisition et d'administration foncières de même qu'un plus grand potentiel de développement agricole capitaliste, il en est autrement en pratique. En effet, de nettes tendances à la monopolisation et à la spéculation foncière marqueront rapidement le développement du territoire tenu en franc et commun soccage au Bas-Canada, produisant indéniablement des effets similaires à ceux créés par la tenure seigneuriale. Elles aussi entravent le développement agricole et limitent l'investissement dans la sphère de production, permettent l'extorsion de surtravail paysan et concourent à la dépossession/expropriation des populations campagnardes. Bref, loin d'activer la pénétration du capitalisme

43 Voir Marta Harnecker, Les concepts élémentaires du matérialisme historique (Bruxelles, Contradictions, 1974), 125-127. 
dans la formation sociale, la tenure soccagère s'insère parfaitement dans le cadre féodaliste dominant. Au niveau des résultats pratiques, elle n'entre aucunement en contradiction avec la tenure seigneuriale et opère en réalité sur la base de rapports d'exploitation de même nature. Elle sert et remplit, à toutes fins utiles, les mêmes objectifs et fonctions socio-économiques.

\section{a - La formation des monopoles}

Par suite de la Conquête, certains mécanismes contribuent à la formation d'un groupe de grands propriétaires fonciers. Au premier chef, on retrouve le patronage et le favoritisme de l'État. Parmi les bénéficiaires des largesses de l'administration coloniale se trouvent d'ex-officiers militaires et des fonctionnaires qui souvent regagnent la Grande-Bretagne tout en conservant leur titre de propriété au Canada.

En second lieu, il y a la distorsion du système dit des «leaders and associates» en vigueur entre 1791 et $1826^{44}$. Théoriquement, cette pratique devait encourager un certain nombre de colons à s'établir sous la direction d'un chef de canton à l'intérieur d'une localité donnée. Dans la pratique cependant, plusieurs chefs de cantons obligeaient les associés à leur remettre 1000 acres sur les 1200 qui leur étaient concédées afin qu'ils puissent se rembourser des frais encourus et des dépenses futures. Par la suite, certains chefs de cantons dressèrent même des listes fictives d'associés et acquirent ainsi la propriété exclusive de vastes portions de territoires. Entre 1799 et 1803, pas moins de 1425000 acres avaient été distribuées à 60 personnes tout au plus ${ }^{45}$.

En 1818, on tenta de remédier à ces abus en introduisant le système des billets de location par lequel un individu n'obtiendrait le titre de propriété sur son lot de 200 acres qu'au moment où quatre acres de terroir seraient défrichées et qu'une maison serait construite. Là encore, spéculateurs et monopolistes trouvèrent le moyen de contourner le système grâce à la complicité des administrateurs coloniaux. Ils engagèrent de la main-d'oeuvre pour défricher les quatre acres requises et faire ériger une cabane en bois rond. Par ce stratagème, un nombre indéfini de lots de 200 acres pouvait être acquis ${ }^{46}$.

En 1825 , le Colonial Office tenta à nouveau de palier à cette situation en instituant un système de distribution foncière qui

44 A. Shortt et A.G. Doughty (sous la direction de), Canada and its Provinces (Toronto, Glasgow, Brook and Co., 1914), 4, section II, partie II: 559-560.

45 Ibid.: 560 et F. Ouellet, Le Bas-Canada..., 57.

46 A. Shortt et A.G. Doughty, op. cit., 560-561. 
devait théoriquement faire obstacle à toute tentative de monopolisation. En vertu de ce système - connu comme le New South Wales System - aucun lot ne devait s'étendre sur plus de 1200 acres. On pouvait acquérir ces lots de la Couronne seulement par achat aux enchères; le colon devait donner, comme premier versement, le quart de la valeur du terrain et s'acquitter du reste de la dette en trois annuités plus 5\% d'intérêt (Quit Rents). Le nombre des terrains mis en vente était déterminé par le gouverneur. L'équité apparente de ce système allait rapidement faire place à des pratiques plus douteuses. On en modifia bientôt les règlements, atténuant de ce fait les quelques avantages apparents qu'il pouvait procurer au colon éventuel: des instructions émanant du Trésor britannique ordonnèrent par la suite l'augmentation du prix de base des terrains, le paiement immédiat des intérêts non payés sur les terres déjà vendues et le paiement intégral du prix des terres au moment même de l'achat ${ }^{47}$. En outre, l'imposition de ce système de distribution foncière, supposément plus équitable, n'arrêta pas le favoritisme qui avait depuis toujours prévalu dans la concession des terres. Alors qu'entre 1825 et 1831, 450469 acres avaient été distribuées conformément au système de vente aux enchères, 641639 acres avaient été livrées gracieusement aux spéculateurs par la Couronne pendant la même période ${ }^{48}$. Ce qui plus est, certains gouverneurs s'opposaient même à l'application du New South Wales System, arguant que les colons éprouvaient de la difficulté à effectuer leurs paiements. En réalité, l'administration coloniale dédaignait ce système de distribution foncière qui, potentiellement, pouvait l'empêcher de poursuivre librement sa politique de favoritisme à l'égard des éléments influents de la colonie susceptibles de lui accorder leur appui ${ }^{49}$.

Il devient évident que la spéculation sur les terres ne fut jamais sérieusement menacée. Les intéressés parvenaient toujours à contourner la loi et à poursuivre impunément leurs pratiques foncières monopolistes. Par suite de toute cette activité qui avait cours depuis la Conquête, il restait peu de terres disponibles vers 1830 , soit au moment précis où les besoins atteignaient des sommets (pressions démographiques en territoire seigneurial et arrivée de milliers d'immigrants britanniques). En réalité, les terres étaient disponibles, mais elles n'étaient pas accessibles. Les individus à qui l'on avait si généreusement distribué de vastes portions de territoi-

47 C.D. Smith, The Role of Land Alienation, Colonization and the British American Land Company on Quebec's Development 1800-1850, thèse de M.A. (sociologie) (Université McGill, 1974), 149.

48 Idem.

49 Ibid, 150. 
re $^{50}$ devaient théoriquement prendre les moyens pour encourager et promouvoir la colonisation sur ces terres. Ils n'en faisaient rien et le gouvernement fermait les yeux. Pas plus qu'il ne se préoccupait de la spéculation exercée par les propriétaires absentéistes qui freinaient la colonisation des Cantons de l'Est. En 1838, par exemple, seulement 6 propriétaires sur les 105 détenant 1300000 acres dans les Cantons de l'Est, vivaient sur leur propriété ${ }^{51}$. Au surplus, chaque canton devait réserver $2 / 7$ de sa superficie: $1 / 7$ demeurait propriété de la Couronne pour être vendu plus tard et l'autre 1/7 allait à l'Église anglicane. Ces réserves à elles seules pouvaient couvrir vers 1835 près de 1750000 acres. La part de l'Église anglicane s'élevait en 1838 à 919013 acres ${ }^{52}$.

Dans ce contexte, lorsqu'un grand propriétaire acceptait de vendre quelques terrains, c'était le plus souvent à un prix exhorbitant que très peu de paysans, même parmi les plus aisés, pouvaient se permettre. En outre, les colons devaient subir tellement de tracasseries administratives pour obtenir la moindre parcelle de terre de la Couronne ou du Clergé que nombreux étaient ceux qui abandonnaient tout projet d'établissement agricole. Dépossédés, ils descendaient vers les États-Unis ou végétaient tout simplement comme journaliers autour des villes et des seigneuries.

\section{$b$ - La British American Land Company}

La British American Land Company (BALC) représente un des cas les plus évidents d'aliénation et de monopolisation foncières et ne fait que maintenir une tradition fortement enracinée. L'exemple de cette compagnie est d'autant plus pertinent pour notre propos que des éléments de la bourgeoisie marchande bascanadienne s'y trouvent mêlés.

50 On estime que vers 1837 , trois millions et demi d'acres, soit près de la moitié du territoire arpenté de la province, avaient été offertes à quelques-uns à titre de gratification ou récompenses politiques. (W.H. Parker, «A New Look at Unrest in Lower Canada in the 1830's», Canadian Historical Review, 40 (1959): 212). Gary Teeple explique la situation sans équivoque: "Political favourites, merchants, and a coterie of colonial officials from the governor to the land surveyor were among the land speculators of the time. Very little of the land was developed or opened for settlement, as the speculators were interested only in the profit they could make from the rising prices of land as immigration increased. The monopolization of such vast lands by so few was clearly a deterrent to settlement (G. Teeple, "Land, Labour, and Capital in Pre-Confederation Canada», in G. Teeple (sous la direction de), Capitalism and the National Question in Canada (Toronto, University of Toronto Press, 1972), 49).

51 C.D. Smith, op. cit., 141.

52 Gérard Filteau, Histoire des Patriotes (Montréal, L’Aurore, 1975), 59. 
En 1825, des financiers britanniques et leurs associés montréalais ${ }^{53}$ proposèrent d'acheter toutes les terres non concédées dans les cantons arpentés du Bas-Canada, plus la moitié ou la totalité des réserves du clergé au prix en vigueur avant mars $1824^{54}$. Lord Dalhousie, alors gouverneur de la province, s'y opposa. Il invoqua diverses raisons dont l'arpentage inadéquat du territoire provincial, l'état encore non définitif des frontières des cantons et des comtés, l'impossibilité de vendre les terres seigneuriales déjà possédées, étant donné l'état de confusion dans certains titres de propriété et, enfin, une certaine incrédulité chez lui quant aux possibilités des investisseurs de se procurer la somme exigée ${ }^{55}$.

Néanmoins, en 1832 , ces promoteurs refont surface et réitèrent leur volonté d'acquérir de vastes portions de territoire à des fins de développement. Décrivant leurs objectifs comme étant de nature purement philanthropique et se disant prêts, par surcroît, à financer l'émigration de colons en provenance des îles britanniques, ils parviennent à circonvenir les autorités impériales et coloniales en mettant en relief la solution que constituerait la formation d'une telle compagnie aux problèmes créés par les misérables conditions de vie du prolétariat britannique. Incorporée en mars 1834, la Compagnie sera confirmée dans son existence par un Acte du Parlement anglais en mai 1834. Elle peut, dès lors, acquérir 847611 acres dans les Cantons de l'Est pour la somme de $£ 120000$ payables en dix ans avec intérêt de $4 \%$ - conditions de crédit vraisemblablement plus avantageuses que celles imposées par le New South Wales System. Ce prix incluait en outre l'appropriation des droits d'exploitation forestière et minière sur la totalité des territoires achetés.

La Compagnie s'engageait à favoriser l'établissement des immigrants britanniques sur ces terres en construisant les immeubles nécessaires à l'organisation socio-économique des cantons (moulins, boulangeries, forges, magasins, etc.). Toutefois, il est permis de mettre en doute cette magnanimité; non seulement la Compagnie bénéficiait-elle d'un actif de près de $£ 300000$ et de conditions avantageuses de paiement, mais dans plusieurs cas, elle installait les nouveaux colons près de villages déjà existants, de sorte qu'elle minimisait substantiellement ses dépenses d'infrastructure. Enfin, elle revendait les terrains à des prix variant entre 5

53 D. Creighton rapporte qu'au nombre des associés canadiens figuraient les noms de George Moffat et Peter McGill, lesquels agissaient en tant que commissaires résidents pour la compagnie. (D.G. Creighton, The Empire of the St-Lawrence (Toronto, MacMillan, 1970), 276-277).

54 C.D. Smith, op. cit., 158.

55 Norman McDonald, Canada 1763-1841. Immigration and Settlement (Londres, Longman, Green, 1939), 288. 
et 7 shillings l'acre pour les terres en friche et entre 10 et 20 shillings l'acre pour les terres défrichées. Elle réalisait ainsi des profits variant de $150 \%$ à $650 \%$ sur ces terres, puisqu'elle ne les avait payées que 3 shillings l'acre. Après une année d'activité seulement, elle peut étendre son avoir foncier à 1094272 acres $^{56}$.

Il appert en fait que l'établissement dans les cantons par l'intermédiaire de la Compagnie des terres ne fut qu'une grande entreprise de spoliation foncière et d'agiotage à l'avantage de la bourgeoisie marchande d'ici, des financiers britanniques et de plusieurs administrateurs coloniaux. En tant qu'entreprise de colonisation, elle ne connut qu'un succès limité ${ }^{57}$. Cela est en grande partie imputable aux modalités de paiement de la terre convoitée. Seul le colon qui acquitte le paiement entier de son achat acquiert sur le champ son titre de propriété. Peu d'entre eux disposent de la somme nécessaire. Pour les autres, l'achat se fait à tempérament et la Compagnie conserve jusqu'à liquidation de la dette la propriété de la terre. Le colon verse $20 \%$ du coût d'achat et acquitte le solde par versements. Durant les quatre premières années, il ne paie que l'intérêt sur le capital, tandis qu'au cours des quatre années suivantes, il doit rembourser le capital. Bref, ce n'est qu'au bout de neuf ans - si tout va bien - que le colon acquiert son titre de propriété $^{58}$. Plusieurs des nouveaux colons pouvaient difficilement rencontrer ces exigences de paiement et accumulaient ainsi des arrérages. En conséquence, ils étaient éventuellement dépossédés, le plus souvent après qu'ils eussent entrepris quelques travaux sur leur lot. En d'autres occasions on les forçait à payer leurs arrérages en exigeant d'eux qu'ils travaillent pour le compte de la Compagnie à ouvrir des chemins ou à réaliser d'autres besognes similaires. Ils payaient de cette manière souvent plus qu'ils ne devaient en réalité sans garantie de droits éventuels de propriété. On les menaçait en outre de confisquer leur bien foncier s'ils ne participaient pas à ces travaux ${ }^{59}$.

56 C.D. Smith, op. cit., 160-161.

57 Le succès de l'entreprise ne fut pas à la mesure de ce que l'on avait escompté. L'opposition farouche de la Chambre d'Assemblée à son égard, le manque de capitaux des immigrants, les difficultés de coloniser des Cantons situés derrière des seigneuries et au milieu des domaines non cultivés des spéculateurs fonciers firent qu'à sa quatrième année d'existence, la Compagnie ne put ou ne voulut rencontrer ses obligations financières envers l'administration. Le gouvernement reprit en 1840 la moitié du territoire concédé à la compagnie. Cette dernière conserva plus de 600000 acres parmi les mieux situées. Le bilan net de l'aventure fut d'ajouter un grand propriétaire de plus au nombre déjà impressionnant que comptait le Bas-Canada et de rendre ainsi encore plus difficile l'accès à la petite propriété foncière. (Maurice Séguin, La nation canadienne et l'agriculture, 1760-1850 (Trois-Rivières, Boréal Express, 1970), 200-201).

58 C.D. Smith, op. cit., 163.

59 «Premier rapport du Comité permanent sur les Terres et Droits seigneuriaux», in JCABC, 1835-1836, appendice EEE. Témoignage de Robert Layfield. 
Ces pratiques de la BALC s'inscrivent en fait dans le prolongement d'une longue tradition d'entrave à la colonisation. Elles s'ajoutent aux méthodes frauduleuses de distribution foncière sanctionnées et mises en oeuvre par l'administration coloniale ellemême. Ainsi, il était souvent arrivé que des fonds publics ou des octrois fonciers soient détournés et utilisés à des fins autres que celles prévues. Un témoin du comté de Mégantic rapporte qu'en 1828, l'agent de la Couronne chargé des immigrants obtenait un octroi de terres et de sites de moulin afin d'y ériger des moulins au profit des colons. À l'encontre de ses obligations, il vendit les sites de moulin et établit les colons sur des terres sans grande valeur agricole. Ce même témoin atteste aussi que les deniers de la législature furent employés à construire des chemins sur de grandes étendues de terres inhabitées afin d'en augmenter la valeur, alors que cet argent aurait dû servir au développement des localités où résidaient déjà des colons ${ }^{60}$.

On peut parler dans la réalité de collusion entre les autorités et les spéculateurs privés au point qu'il devient difficile de les distinguer. Dans Mégantic, par exemple, on dit que les trois quarts du comté appartiennent à des officiers publics et chefs de cantons qui théoriquement doivent veiller à la distribution et au développement des terres; en pratique, bien peu sera réalisé. Cette situation semble d'ailleurs généralisée à l'ensemble des cantons. La plupart des individus à qui la Couronne avait concédé de vastes étendues de terres - administrateurs coloniaux ou autres personnages liés à l'administration coloniale - n'avaient que très peu amélioré l'état de leurs concessions au cours des années en dépit de leur obligation en ce sens. Certains n'y avaient même rien fait, ni concession, ni développement ${ }^{61}$. Le temps venu, il était donc dans l'intérêt de ces gens de soutenir la création d'une compagnie des terres. Celle-ci, dotée d'un impressionnant capital, chercherait à élargir son domaine. Les détenteurs de concessions étaient en fait trop heureux de vendre à profit leurs terres à la compagnie plutôt que d'avoir à s'engager à leurs propres frais dans les coûts prohibitifs de la colonisation. D'autant plus que la valeur de leurs terres était souvent augmentée par des colons qui prenaient le risque de s'établir (phénomène des squatters) en espérant qu'on leur cèderait éventuellement la propriété d'un lot. Mais la plupart du temps, au bout de quelques années, les propriétaires les chassaient sans offrir

60) Idem.

61 Dans son témoignage au Comité permanent sur les Terres et Droits seigneuriaux, Amury Girod présente une liste de tous ceux à qui de larges concessions ont été octroyées et dresse le bilan des travaux qu'ils y ont réalisés. Cette liste permet de constater la faible proportion de terres qui furent développées. 
aucun dédommagement; ils avaient désormais le champ libre pour spéculer sur la valeur ajoutée par le travail des colons ${ }^{62}$.

Les diverses pratiques de gestion des terres régies par la tenure soccagère permettent tout autant que le régime seigneurial le maintien de rapports de production non capitalistes. En théorie, cette tenure est censée offrir la propriété absolue (donc, capitaliste). Dans les faits cependant, l'endettement paysan créé par les pratiques foncières permet une extorsion du surtravail sur une base non capitaliste. En effet, comme nous l'avons mentionné, pour rembourser sa dette, le paysan participe souvent sans rémunération aux travaux d'infrastructure (construction de routes, moulins, ponts, etc.) liés à l'amélioration du domaine des grands propriétaires fonciers, qu'il s'agisse d'individus ou d'une entreprise telle la BALC. Dans d'autres cas, le paysan s'expose à une reprise de possession par le propriétaire pour défaut de non-paiement sans compensation pour les améliorations apportées. De manière générale, les pratiques de la BALC par exemple ne vont pas sans susciter un vif mécontentement parmi les populations des cantons. Dans les Cantons de l'Est, en particulier, principal centre de ses opérations, la compagnie des terres était perçue par plusieurs comme une grande source de malheurs et de violation des droits des cultivateurs. Un témoin du canton de Kingsey (Drummond) rapporte en effet que bien que les opinions soient divisées, "la majorité, qui compte un grand nombre de personnes d'origine britannique, est contre cette compagnie, parce que, composée comme elle l'est, d'actionnaires qui résident en Angleterre, tout l'argent qui provient de la vente des terres sort du pays où il devrait être dépensé» ${ }^{63}$. Certains habitants du comté de Stanstead vont même jusqu'à demander en 1836 l'abolition de la Charte de la British American Land Company auprès de la Chambre d'Assemblée, invoquant

62 On retient entre autres le cas de 3 cultivateurs du Canton de Leeds dans Mégantic qui, sur instruction de l'Agent de sa Majesté pour les Émigrés, s'établirent en 1829 et 1830 sur des lots qui faisaient alors l'objet d'un litige entre le gouvernement et le Seigneur de Ste-Croix. Ayant reçu l'assurance que quoi qu'il advienne ils pourraient quand même devenir propriétaires desdits lots, ils se mirent en frais de les développer et de s'y construire. $\mathrm{Au}$ bout de quelques années, alors qu'ils voulaient s'assurer de leur droit de propriété, on devait les informer que des lettres patentes avaient été rédigées en faveur de l'honorable A.W. Cochrane (conseiller exécutif greffier en loi du Conseil législatif et auditeur des lettres patentes) qui les sommaient dès lors de vider les lieux sans leur accorder aucune indemnité. Dans leur pétition à la Chambre d'Assemblée, ces 3 cultivateurs disent avoir «tout lieu de croire que le Bureau des terres a connivé dans cette transaction honteuse, et qu'on a permis audit A.W. Cochrane de remettre à la Couronne certains lots de terres incultes qui lui appartenaient en vertu des lettres patentes, afin qu'on pût donner audit A.W. Cochrane l'occasion de s'emparer du peu de bien que possédaient les pétitionnaires». (Pétition de $\mathrm{J}$. Nevill, C. Higgins et J. Mc Callum, JCABC, 20 janvier 1836, 373).

${ }_{63}$ Premier rapport du Comité permanent sur les terres et droits seigneuriaux, op. cit., témoignage de Calvin Alexander. 
qu'ils considèrent l'octroi d'une Charte à la Compagnie des Terres Britannique Américaine comme une violation de leurs droits et de leurs privilèges comme Sujets Britanniques, qui leur ont été garantis par un Acte du Parlement Impérial: Que les Pétitionnaires regardent de plus la vente d'une aussi grande étendue de Terres publiques, comme une mesure dont les conséquences sont très préjudiciables au bien-être et à la prospérité des Habitants de cette Province, et qui continueront de se faire sentir, non seulement à la présente génération, mais encore avec une force croissante à leur postérité la plus reculée: Que les Pétitionnaires et les Habitants de la Province sont pour la plupart agriculteurs, et ils dépendent de leurs terres pour leur subsistance: Que ce n'est donc pas avec peu d'alarme qu'ils voient une Compagnie étrangère parmi eux, dont l'établissement obscurcit les plus brillantes espérances qu'ils avaient devant eux. ${ }^{64}$

L'existence de compagnies des terres affecte et menace donc également les populations rurales dont la survie économique est reliée à l'expansion du territoire agricole, et ce, peut-on penser, indistinctement de l'origine ethnique, contrairement aux vues de certains historiens ${ }^{65}$.

\section{Conclusion}

Nous avons suggéré dès le départ la place centrale qu'occupe la propriété foncière comme facteur d'organisation socioéconomique de la formation sociale bas-canadienne avant 1846 . Nous avons alors proposé de nous adonner à une relecture de la réalité sociale bas-canadienne à partir de ce foyer d'analyse. Notre étude de la situation foncière a permis de circonscrire quelques paramètres essentiels à la compréhension des caractéristiques structurelles de la formation sociale bas-canadienne de l'époque.

En corollaire, nous avons d'abord constaté que dans le domaine foncier, le clivage le plus important est d'abord d'ordre économique. Au-delà de l'origine ethnique, il oppose propriétaires et non-propriétaires des moyens de production. Deuxièmement, il n'y a pas d'adhésion ethniquement et culturellement motivée à une tenure particulière. Toutefois l'aspect le plus essentiel de cette relecture suggère que, de par les pratiques qui les sous-tendent, les tenures seigneuriale et soccagère se conjuguent pour favoriser, au cours de la période étudiée, la reproduction/domination des structures non capitalistes de la formation sociale. En effet, l'appropriation réalisée par les grands propriétaires fonciers du surtravail

${ }^{64}$ Pétition d'habitants du comté de Stanstead, Journaux de la Chambre d'Assemblée du Bas-Canada, 23 janvier 1836.

${ }_{65}$ Voir entre autres, M. Séguin, op. cit. 
prélevé de manière directe sous forme de rentes et de manoeuvres économiques spoliatrices, et de manière indirecte à travers la tyrannie juridique qui légitime leurs exactions et condamne les paysans à travailler gratuitement pour leur profit, est fondamentalement caractéristique d'un rapport de production de nature féodale. Ce rapport de production marque de façon dominante à tous les niveaux l'orientation générale de la formation sociale bascanadienne.

Notre analyse du mercantilisme au Bas-Canada et des pratiques économiques spécifiques de ses agents nous a permis de mettre à jour les composantes caractéristiques d'une économie marchande. Forme de production insolite s'il en est, elle revêt certaines allures capitalistes, mais procède d'une logique fondamentalement féodaliste. En effet, le capital marchand et ses agents ne s'inscrivent pas dans une espèce de modernité post-féodale, mais s'intègrent parfaitement aux structures féodalisantes du régime seigneurial. De même, dans ses résultats concrets, la spéculation foncière ne contredit en rien les principes directeurs de l'organisation féodale de la société.

L'apparence de rationalité capitaliste de certaines activités de la bourgeoisie marchande dans la sphère agricole s'estompe dans la mesure où elles sont réalisées sur la base de l'extorsion féodale du surtravail paysan et contribuent au maintien de la dominance de rapports de production de nature féodale.

En somme, l'activité marchande qui régit la structure économique bas-canadienne avant 1846 ne relève pas du capitalisme. Il n'y a pas de mode de production capitaliste à proprement parler avant que le capital ne s'empare définitivement de la production. Or, l'accumulation réalisée sous forme de profit marchand et par l'accaparement de revenus seigneuriaux et de spéculation constitue simplement une richesse d'argent et n'est pas transformée en capital. En effet, la bourgeoisie marchande, loin d'encourager le développement d'un secteur productif, défavorise au contraire, par ses pratiques économiques particulières, l'établissement de manufactures ou d'industries sur une vaste échelle. De plus, elle étouffe l'épanouissement d'un marché intérieur en le soumettant - lorsqu'elle ne l'ignore pas totalement - aux impératifs exclusifs du monopole commercial impérial ${ }^{66}$.

\footnotetext{
66 La bourgeoisie marchande bas-canadienne liée au marché impérial s'est toujours inquiétée des tendances libre-échangistes qui se manifestaient ici et en Grande-Bretagne. Loin de penser en termes d'une plus grande ouverture commerciale ou d'investissement dans la sphère de production, elle demandait aux autorités impériales des tarifs préférentiels toujours plus généreux. C'est dans cette optique d'ailleurs que certains de ces représentants mettaient en garde la métropole contre la possibilité d'un développement autonome des potentialités économiques et manufacturières des colonies: «Unless the colonies were
} 
Pourtant, la bourgeoisie marchande bas-canadienne contribue à l'induction de tendances capitalistes appelées à se déployer pleinement après 1846 au sein de la formation sociale et ce, de trois façons: d'abord en réalisant une certaine accumulation de capital, ensuite en contribuant par ses pratiques foncières à la prolétarisation d'une partie de la paysannerie ${ }^{67}$ et finalement par les retombées engendrées par le commerce du bois au sein de la sphère de production ${ }^{68}$. Ce dernier aspect n'a pas été développé dans le cadre de ce texte. Cependant, il faut réaliser que bien que dominante, l'économie marchande et le rapport de production qui la caractérise n'en occupent pas pour autant la totalité de l'espace économique. On décèle en effet l'existence de formes économiques capitalistes qui viennent se juxtaposer aux formes économiques marchandes et qui restent dominées durant la période considérée. La démonstration de cette facette de la structure économique bascanadienne dépasse notre propos. Des recherches en cours nous permettent toutefois d'avancer l'hypothèse que la bourgeoisie marchande ne tente pas vraiment au cours de cette période d'intégrer à ses activités les potentialités manufacturières de la colonie. En fait, ces tendances capitalistes se manifestent plus particulièrement par l'expansion de la sphère de la petite production et par l'introduction en son sein de nouveaux rapports de production. Cette expansion se traduit par un passage graduel de l'artisanat à la manufacture. Le procès de travail et les rapports afférents à l'artisanat cèdent le pas à une division sociale du travail plus poussée et à des rapports de production plus typiquement capitalistes ${ }^{69}$.

encouraged to devote thernselves exclusively to the production of agricultural produce and of other raw materials, they would undoubtedly turn their attention to manufacturing before long, and attempt to pass laws for its protection from outside competition.» (Mémoire de la North American Committee of the Colonial Association, cité dans Adam Shortt, «Economic History, 1840-1867» dans A. Shortt and A.G. Doughty (eds.), Canada and its Provinces, 5, section III, United Canada (Toronto, Glasgow, Brook and Co., 1914): 196.

67 Pour des détails à propos de la réalisation de ce processus, voir Gérald Bernier, "Sur quelques effets de la rupture structurelle engendrée par la Conquête au Québec», Revue d'histoire de l'Amérique française, 35, 1 (juin 1981): 81-89.

68 La généralisation de l'économie marchande et l'expansion du commerce du bois en particulier sont dans une large mesure responsables des retombées économiques dans la sphère de production. Ainsi, de nombreuses entreprises sont directement subordonnées au commerce du bois: les scieries et manufactures de bois de construction, les chantiers de construction navale et les chantiers de construction immobilière. D'autres répondent aux besoins de consommation courante des populations attirées par l'activité commerciale vers les villes et leur périphérie immédiate: distilleries, brasseries, fabriques de chapeaux, tanneries, etc. Enfin, une dernière catégorie d'entreprises spécialisées dans la fabrication de machines-outils, d'engins à vapeur (fonderies) satisfait aux besoins d'outillage et de machinerie nécessités par les divers chantiers de construction navale et l'exportation forestière en particulier. Voir Robert Tremblay, La nature du procès de travail à Montréal entre 1790 et 1830, mémoire de maîtrise (histoire) (Université de Montréal, 1979), 43-51.

69 Voir ibid et Daniel Salée, Transition, luttes et alliances de classes: Une analyse du mouvement révolutionnaire de 1837-38 au Québec. Mémoire de maîtrise (science politique) (Université de Montréal, 1980), 146-158. 
À la décharge de la bourgeoisie marchande, il faut souligner que les impératifs du lien colonial dont elle est si étroitement dépendante, ne sont pas étrangers à son manque de dynamisme au sein du secteur productif. La Grande-Bretagne avait élaboré une série de mesures qui restreignaient la liberté de commerce soit avec des pays étrangers, soit avec d'autres colonies britanniques, s'assurant ainsi un monopole commercial. Par ailleurs, ce qui est encore plus significatif, d'autres dispositions empêchaient la fabrication au sein des colonies de plusieurs catégories de biens, l'exportation vers les colonies de certaines machineries industrielles et l'émigration de certains travailleurs spécialisés ${ }^{70}$. Le développement interne d'une métropole, on le sait, s'effectue le plus souvent au détriment des régions périphériques qu'elle contrôle. Pour permettre le maintien de ce rapport d'exploitation et assurer la conservation de sa position monopoliste au sein de la formation sociale coloniale, la métropole doit faire en sorte de réduire ou de minimiser les surplus économiques de la périphérie et immobiliser le plus longtemps possible ses rapports de production au moyen de pressions extra-économiques archaïques. Ce faisant, la métropole doit pouvoir retarder tout processus de différenciation sociale et l'expansion des marchés à l'intérieur de la formation sociale périphérique $^{71}$. Agent de facto des intérêts économiques métropolitains, la bourgeoisie marchande bas-canadienne prône et encourage ce retard, notamment par sa gestion foncière. L'exercice de sa domination économique au Bas-Canada n'aura constitué en réalité qu'une des modalités de développement de la structure économique britannique.

Les éléments présentés ici, s'ils sont valides, devraient permettre d'apprécier sous un jour différent le contexte global de l'époque considérée. En effet, non seulement notre analyse remet-elle en question les idées reçues sur certains aspects de la structure économique bas-canadienne et sur la nature de la bourgeoisie marchande, mais elle impose aussi un prolongement analytique au niveau de l'interprétation sociale globale. C'est à ce niveau -

70 Pour plus de détails sur toutes ces questions voir W.T. Easterbrook et Hugh G.J. Aitken, Canadian Economic History (Toronto, Macmillan, 1970); Adam Shortt, "General Economic History, 1763-1841", in Adam Shortt et Arthur G. Doughty (sous la direction de), Canada and its Provinces, vol. 4, section II, British Dominion, partie II (Toronto, Glasgow, Brook and Co., 1914), 521-596; D.C. Coleman, The Economy of England, 1450-1750 (Londres, Oxford University Press, 1977); et les trois ouvrages de Peter Burroughs, The Colonial Reformers and Canada, 1830-1849 (Toronto, McClelland and Stewart, 1969); British Attitudes towards Canada, 1822-1849 (Scarborough, Prentice-Hall of Canada, 1971); The Canadian Crisis and British Colonial Policy 1828-1841 (Toronto, MacMillan of Canada, 1972).

71 Ernesto Laclau, «Feudalism and Capitalism in Latin America», dans E. Laclau (ed.), Ideology, Politics and Marxism (Londres, NLB/verso, 1977), 37. 
essentiel dans notre optique - que se situent nos recherches actuelles.

Elle peut, par exemple, éclairer certains aspects du vécu sociopolitique bas-canadien. Ainsi, dans la mesure où la bourgeoisie marchande n'apparaît plus comme une classe capitaliste au sens propre, on peut mieux saisir sa manipulation quasi-absolutiste des institutions juridico-politiques qui, en dépit de leur caractère libéral et démocratique bourgeois, ne servent, le plus souvent, qu'à accentuer l'inféodation socio-économique des masses paysannes. La reproduction des structures économiques féodales de la société orchestrée de facto par la bourgeoisie marchande peut expliquer son conservatisme politique, son mépris du processus parlementai$\mathrm{re}^{72}$, et les raisons pour lesquelles elle cherchera à plusieurs reprises à contracter alliance avec les membres de l'ancienne aristocratie francophone dont l'influence idéologico-politique était encore solide dans plusieurs régions.

Nous croyons qu'en insistant dans un premier temps sur les clivages d'ordre socio-économique et en leur donnant dans l'analyse toute la place qu'il convient, il devient possible d'entrevoir les axes de conflits selon une perspective globale de compréhension des antagonismes sociaux qui prennent forme à tous les niveaux structurels de la formation sociale bas-canadienne. Il s'agit en cela de permettre à l'analyse socio-politique de la période de dépasser les confins étroits d'une démarche analytique liée presque exclusivement au problème apparemment posé par la dualité ethnique ${ }^{73}$.

Bref, il faut voir dans notre contribution une étape préliminaire vers une compréhension nouvelle de l'histoire et de l'économie politique du Bas-Canada. Une compréhension basée d'abord et avant tout sur la configuration structurelle qui marque alors le développement particulier de la société québécoise et non pas sur les a priori subjectifs d'une quelconque problématique culturaliste. C'est en ce sens qu'il faudra orienter les réflexions ultérieures.

72 Un des plus bels exemples de ce mépris se trouve dans l'usage que la bourgeoisie marchande fera du Conseil législatif. Ce corps gouvernemental nommé par le gouverneur s'est souvent arrogé des pouvoirs discrétionnaires et exécutifs qu'il n'avait pas légalement. Ainsi, en 1832, il fit emprisonner les éditeurs des journaux La Minerve et The Vindicator parce qu'ils l'avaient critiqué publiquement. De façon générale, il usait aussi très souvent de son veto législatif pour débouter les projets de lois soumis par la Chambre d'Assemblée. (Pour des exemples de son action dans le domaine foncier, voir note 42).

73 Pour un aperçu de l'optique analytique à laquelle nous adhérons, voir Daniel Salée, Fétichisme, analyse historique et la question nationale: Le cas des insurrections de 1837-1838 au Québec (Montréal, Collection «Notes de recherche», Département de science politique, Université de Montréal, décembre 1981). 